\title{
Decreased Physical Activity Attributable to Higher Body Mass Index Influences Fibromyalgia Symptoms
}

\author{
Ann Vincent, MD, Daniel Clauw, MD, Terry H. Oh, MD, Mary O. Whipple, BA, \\ Loren L. Toussaint, PhD
}

Background: Although previous studies report associations between increased body mass index (BMI) and fibromyalgia symptoms, there is uncertainty as to whether this relationship is driven by physical factors, psychological factors, or both.

Objective: To assess these relationships in a clinical sample of patients with fibromyalgia.

Design: Cross-sectional study.

Setting: Tertiary care facility.

Patients: A total of 686 patients from an existing national fibromyalgia registry.

Methods: Patients completed a demographic form and self-report questionnaires including the Fibromyalgia Impact Questionnaire-Revised (FIQ-R), the Medical Outcomes Study Short Form-36 (SF-36), the Brief Pain Inventory (BPI), and the 30-item Profile of Mood States (30-item POMS).

Main Outcome Measurements: FIQ-R overall impact subscale.

Results: BMI was significantly correlated with fibromyalgia impact $(P<.001)$. The relationship between BMI and fibromyalgia impact was almost fully accounted for by physical factors and not by psychological factors.

Conclusions: Despite patient report that pain hinders physical activity, clinicians who encounter patients with fibromyalgia, particularly patients with increased BMI, should be cognizant of the need to invest time and resources to counsel patients on physical factors (ie, physical activity) that could improve the patients' symptom experience.

PM R 2014;6:802-807

\section{INTRODUCTION}

Body mass index (BMI) above the normal range is a determinant both of more severe symptoms and of worse health outcomes in patients with fibromyalgia [1-3]. Despite the association between BMI and fibromyalgia symptoms, it is unclear whether this relationship is driven by physical factors, psychological factors, or both [2,4-7]. This information is important, as the prevalence of obesity in patients with fibromyalgia is high (47\%-73\%) $[1,5,7]$, and clinicians who encounter patients with fibromyalgia with an unhealthy BMI need clear guidance on the most appropriate management for this group of patients.

Many factors, including decreased physical activity, stress, depression, anxiety, and chronic sleep deprivation, have been linked with body weight in patients with fibromyalgia $[4,7]$. For example, previous research has demonstrated that in fibromyalgia, being obese is associated with greater pain severity, greater number of tender points, poorer sleep quality, reduced physical strength and flexibility, and higher symptom burden when compared to being of normal weight $[2,5,6]$. Also, women with fibromyalgia who are obese have significantly higher levels of anxiety and depression, poorer quality of life, and poorer physical performance than women with fibromyalgia who are not obese [6]. Despite this body of research, no study to date has assessed the complex association between BMI, physical, and psychological variables in the same model.

To evaluate complex relationships between variables, specific statistical techniques such as mediation models that estimate direct relationships between variables and effects of

\begin{abstract}
A.V. Fibromyalgia and Chronic Fatigue Clinic, Mayo Clinic, 200 First Street SW, Rochester, MN 55902. Address correspondence to: A.V; e-mail: vincent.ann@mayo.edu Disclosure: nothing to disclose
\end{abstract}

D.C. Chronic Pain and Fatigue Research Center, University of Michigan, Ann Arbor, MI Disclosures related to this publication: grant (money to institution), Pfizer, Cerephex, Lilly, Merck, Nuvo, Forest; consulting (money to author), Pfizer, Cerephex, Lilly, Merck, Nuvo, Forest, Tonix, Purdue, Therauance, Johnson \& Johnson

Disclosures outside this publication: expert testimony (money to author), Forest, Pfizer; patents (money to author), pain testing device; royalties (money to author), pain testing device

T.H.O. Fibromyalgia and Chronic Fatigue Clinic, Mayo Clinic, Rochester, MN Disclosure: nothing to disclose

M.o.w. Fibromyalgia and Chronic Fatigue Clinic, Mayo Clinic, Rochester, MN Disclosure: nothing to disclose

L.L.T. Department of Psychology, Luther College, Decorah, IA

Disclosures related to this publication: consulting fee or honorarium (money to author), statistical consult fee from Mayo Clinic

This study was supported in part by the Center for Translational Science Activities (CTSA) at Mayo Clinic. This center is funded in part by a grant from the National Center for Research Resources (NCRR), a component of $\mathrm{NIH}(\mathrm{RRO24150)}$. Its contents are solely the responsibility of the authors and do not necessarily represent the official view of the CTSA, NCRR, or $\mathrm{NIH}$.

Peer reviewers and all others who contro content have no relevant financial relationships to disclose.

Submitted for publication October 2, 2013; accepted February 13, 2014. 
mediators can be used [8]. Because our aim was to examine associations among BMI, physical factors, and psychological factors in patients, we created a conceptual mediation model (Figure 1). Specifically, we evaluated whether physical factors (physical activity and physical interference related to pain) or psychological factors (depression and anxiety) arbitrated this relationship. We hypothesized that (1) BMI would be associated with overall fibromyalgia impact, and (2) this relationship would be mediated by depression and anxiety and by physical activity and physical interference from pain.

\section{METHODS}

Data for this analysis were derived from a sample of patients with fibromyalgia $(\mathrm{N}=1303)$ who were randomly selected from a national fibromyalgia registry [9] and were invited to participate in a mailed survey. The overall response rate to this survey was $65.5 \%$. This study was approved by the Mayo Clinic institutional review board.

\section{Participants}

Only those respondents who met Fibromyalgia Research Survey Criteria [10] were included in the present analyses ( $\mathrm{N}=686,52.6 \%$ of the original sample). This is defined as a widespread pain index (WPI) of $\geq 7$ and symptom severity (SS) $\geq 5$ or a $3 \leq$ WPI $\leq 6$ and $S S \geq 9$. In addition, patients who were underweight $(n=15)$ were excluded, as factors responsible for their symptom profiles may be different [4].

\section{Measures}

Participants completed a questionnaire package that included the Fibromyalgia Research Survey [10], the Fibromyalgia Impact Questionnaire-Revised (FIQ-R) [11], the Medical Outcomes Study Short Form-36 (SF-36) [12], the Brief Pain Inventory (BPI) [13], and the 30-item Profile of Mood States (30-item POMS) [14]. For these analyses, we selected specific subscales (described below) to avoid overlap between predictor and outcome variables. For example, the FIQ symptom subscale was avoided, as it shared items with both the BPI and POMS.

$F I Q-R$. The FIQ-R is a 21 -item, validated, self-report measure used to assess symptom burden in patients with fibromyalgia [11]. The FIQ-R yields 3 subscales (functioning, overall impact of fibromyalgia, and symptom severity) and a total summary score. Total scores range from 0 to 100 , and higher scores are indicative of greater symptom burden. The FIQ-R has an internal consistency of 0.95 and is commonly used in clinical trials of patients with fibromyalgia [15-18]. For this analysis we selected the FIQ-R overall impact subscale, which specifically assesses patients' experiences of how overwhelmed they were by fibromyalgia and how having fibromyalgia influenced their ability to accomplish goals. This subscale was selected because it had no conceptual overlap with the predictor or mediator variables.

SF-36. The SF-36 is a 36-item, validated self-report measure that assesses quality of life and burden of disease [12]. It yields 8 subscales (physical functioning, role-physical, bodily pain, general health, vitality, social functioning,

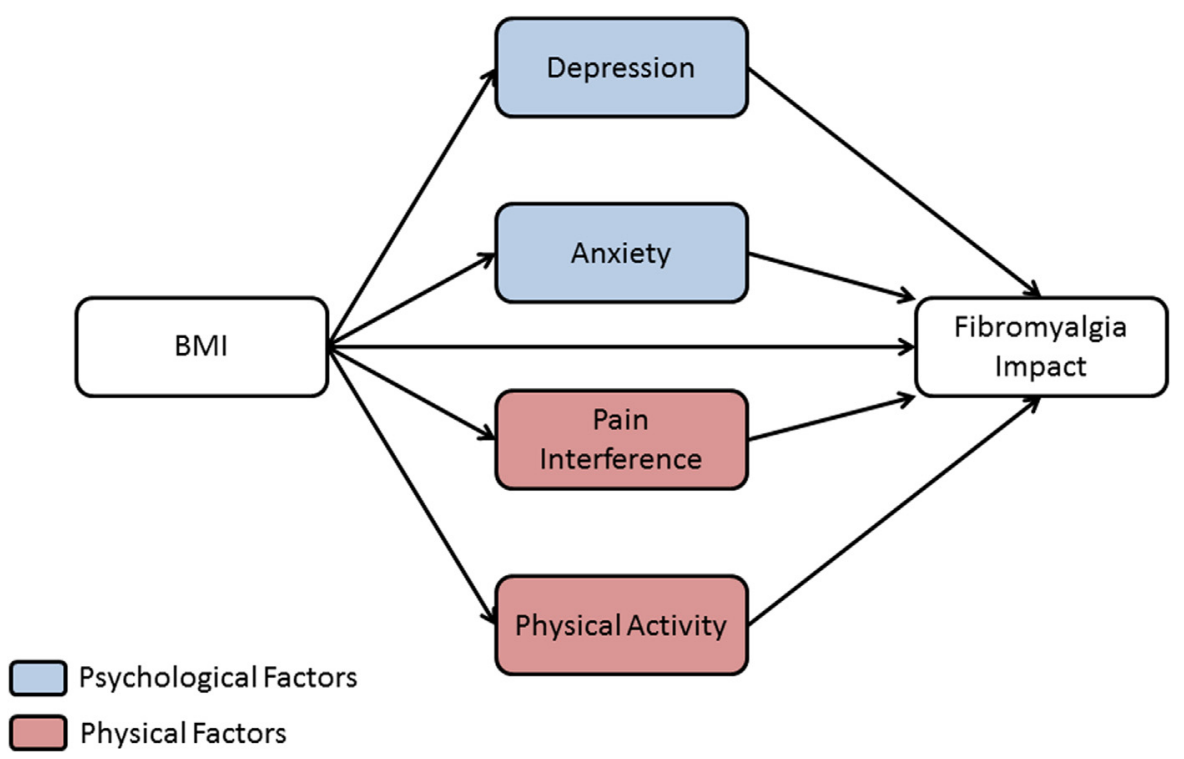

Figure 1. Conceptual model of the association between body mass index (BMI) and fibromyalgia impact, including 4 theoretically derived putative mechanisms. 
role-emotional, and mental health) and 2 summary scores (physical and mental). Component scores range from 0 to 100 , and higher scores are indicative of better health. The SF-36 has an internal consistency of 0.9 and has been used in clinical trials of patients with fibromyalgia [19-21]. For this analysis, we selected the SF-36 physical functioning subscale, which assesses a patient's ability to perform a variety of tasks, including vigorous and moderate activities, walking, bending/kneeling, and bathing/dressing.

$\mathrm{BPI}$. The BPI is a 15 -item, validated self-report measure of chronic pain that assesses the presence of pain, pain severity, and degree of pain interference [13]. The BPI yields 2 subscales: pain severity and pain interference. Scores on the pain severity and pain interference subscales range from 0 to 10 , and higher scores are indicative of greater pain. Internal consistency for the pain severity score is 0.85 and for the interference scale is 0.88 . The BPI has been used in fibromyalgia clinical trials, and is considered an appropriate measure of pain in fibromyalgia [16,22-25]. For this analysis we selected the pain interference subscale, which assesses the degree to which pain restricts or hinders physical activities of daily living.

30-Item POMS. The 30-item POMS is a validated, selfreport measure of mood that yields 6 subscales (depressiondejection, tension-anxiety, fatigue-inertia, vigor-activity, anger-hostility, and confusion-bewilderment) and a total mood disturbance score [14]. Scores on each subscale range from 0 to 20, and higher scores are indicative of more severe symptoms on all scales except for the vigor-activity scale, where lower scores indicate more severe symptoms. The 30-item POMS has an internal consistency of 0.69 to 0.88 and has been used in patients with fibromyalgia [26-28]. For this analysis we selected the depression-dejection and the tension-anxiety subscales to represent the symptoms of depression and anxiety, respectively.

\section{Data Analyses}

Data analyses were conducted using SPSS 19 (IBM Corp., Armonk, NY) and Amos 19 (Amos Development Corp., Crawfordville, FL). Amos 19 was used to estimate structural equation models that tested model fit and total, direct, and indirect effects. Our analyses used guidelines by Kline [29] for model fit, and guidelines by Hayes [30] for assessing direct and indirect effects. Generally accepted cutoffs for model fit in structural equation models are $\geq 0.90$ for the comparative fit index (CFI) and $\leq 0.05$ for the root mean square error of approximation (RMSEA) [29]. SPSS was used to estimate an initial Pearson correlation matrix, to examine individual mechanisms, and to test the statistical significance of each indirect effect using Hayes' Process Macro [30]. Before each analysis, data were examined for adherence to distributional assumptions and outliers. Statistical test results were deemed significant at the $P<.05$ alpha level.

\section{RESULTS}

Overall, 686 patients met inclusion criteria. On average, patients were 56.1 years ( \pm 12.5 years) of age, had a BMI of 30.2 $( \pm 7.3)$, were female $(92.9 \%)$, and were of white ethnicity (90.8\%). Patients exhibited moderate to severe symptom severity as evidenced by a total FIQ-R score of 55.5 ( \pm 19.0 ; range, 0-100). Means and standard deviations for variables included in the mediation model are reported in Table 1.

Pearson correlations (Table 2) demonstrated expected but modest associations between BMI and pain interference $(\mathrm{r}=$ $0.108, P=.005)$, physical activity $(r=-0.280, P<.0001)$, and overall fibromyalgia impact $(r=0.138, P=.0003)$ (Table 1). BMI did not correlate with depression ( $\mathrm{r}=0.050$, $P=.20)$ or anxiety $(r=-0.001, P=.97)$ and hence were not included in mediation analyses. The model tested the mediating effects of pain interference and physical functioning on the BMI-fibromyalgia impact association. Figure 2 provides the results of this model and shows the nonstandardized regression coefficients for each path. This path diagram reveals that a 1-unit increase in BMI results in a small (0.04) increase in pain interference, whereas the same 1-unit increase in BMI results in a moderate $(-0.39)$ decrease in physical activity scores. Likewise, a 1-unit increase in pain interference scores results in a much larger (1.28) increase in fibromyalgia impact scores, and a 1-unit increase in physical activity scores results in a moderate $(-0.17)$ decrease in fibromyalgia impact scores. There was a small, statistically significant, indirect effect of BMI through pain interference and physical functioning on fibromyalgia impact $(B=0.11$, $P=.02$ ). This indicates that a 1-unit increase in BMI acts, through increased pain interference and decreased physical activity, to result in an increase in fibromyalgia impact scores of 0.11. There was no direct effect of BMI on fibromyalgia impact, and removing the direct effect had no adverse effect on model fit $\left[\Delta \chi^{2}(1, N=657)=0.01, P=.97\right]$, which continued to show overall acceptable fit $\left[\chi^{2}(1, \mathrm{~N}=657)\right.$ $=0.03, P=.86 ; \mathrm{CFI}=1.00 ;$ RMSEA $=0.00]$. Using the Process Macro $[30,31]$ for SPSS 19 (SPSS Inc., Chicago, IL), a multiple mediation analysis allowed a test of each indirect effect. Small indirect effects of BMI on fibromyalgia impact acted through both pain interference $(B=0.041, P<.05)$ and physical functioning $(B=0.067, P<.05)$, and these indirect effects were equal in magnitude $(B=0.03, P>.05)$. Finally, a reverse mediation model was examined. In this model, BMI acted as the mechanism of action for associations between depression, anxiety, pain interference, and physical functioning with fibromyalgia impact. This model did not provide a good fit to the data $\left[\chi^{2}(2, N=657)=408.19\right.$, $P<.001 ; \mathrm{CFI}=0.45 ; \mathrm{RMSEA}=0.56]$.

\section{DISCUSSION}

Our results demonstrate that physical factors such as physical impairment related to pain and being physically active 
Table 1. Demographic characteristics and descriptive statistics for questionnaires

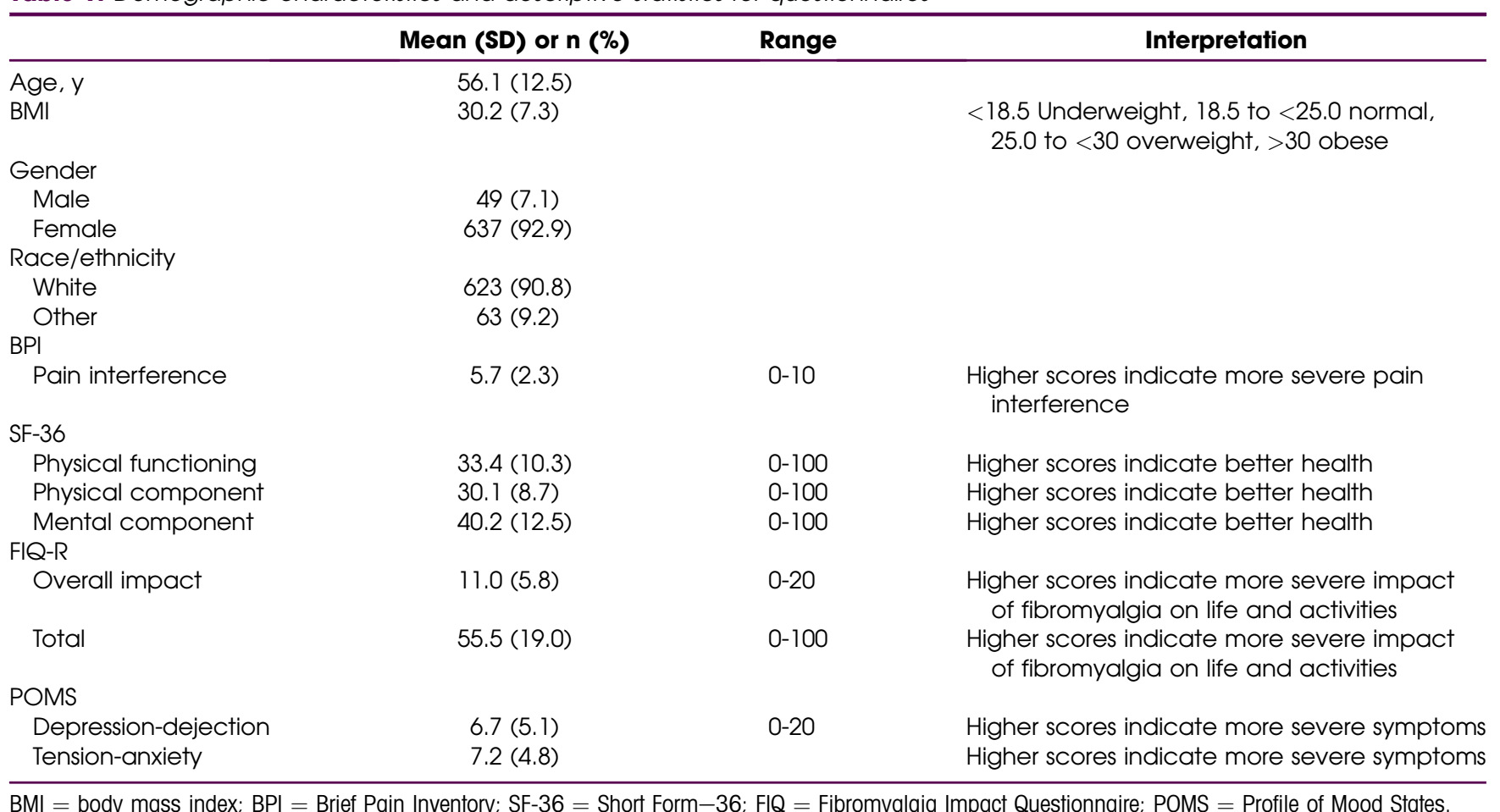

mediate the modest impact of BMI on fibromyalgia symptoms. Depression and anxiety were not correlated with BMI, so they could not explain the relationship between BMI and fibromyalgia impact. Our results are particularly noteworthy, given that ours was a tertiary care population with higher medical and psychological comorbidities, as indicated by SF36 scores that were significantly lower than those of the general population in the United States [12].

Our findings indicate that as BMI increases, it becomes more difficult for a patient to physically function and to be active, which in turn influences the impact of fibromyalgia. Although patients attempt to mitigate the effects of pain by being less physically active, based on our results, lower physical activity actually worsens the impact of the illness. Although vigorous physical activity may not be feasible for patients with chronic pain, physical activity can be increased in multiple ways. For example, Fontaine and Clauw have demonstrated that accumulating 30 minutes per day of lifestyle physical activities (such as walking, using the stairs, gardening) was feasible for previously inactive patients with fibromyalgia, and that this increased physical activity decreased pain [32]. In addition, Levine et al demonstrated that modifying simple lifestyle activities (for example, walking and talking on the telephone instead of sitting, having walking instead of sitting meetings in daily encounters, engaging in purposeful housekeeping, increasing stair climbing) increased non-exercise activity thermogenesis and decreased BMI [33]. Such activities may be pragmatic alternatives to aerobic exercise, and may help patients with fibromyalgia and high BMI to start moving.

Our study has several limitations. First, this is a crosssectional analysis, and correlations do not imply causality; therefore, our results must be interpreted with caution. Only a longitudinal study that assesses temporal relationships of variables over time can shed light on causation. In this study, in addition to correlations, we evaluated the

Table 2. Pearson correlations of body mass index and physical and psychological factors

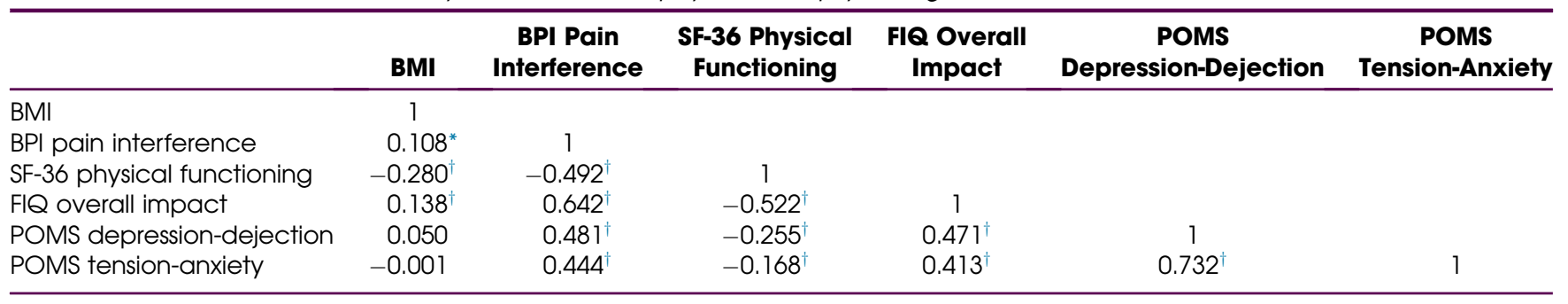

$\mathrm{BMI}=$ body mass index; BPI = Brief Pain Inventory; SF-36 = Short Form-36; FIQ = Fibromyalgia Impact Questionnaire; POMS = Profile of Mood States.

$* P<.01$.

${ }^{\dagger} P<.001$. 


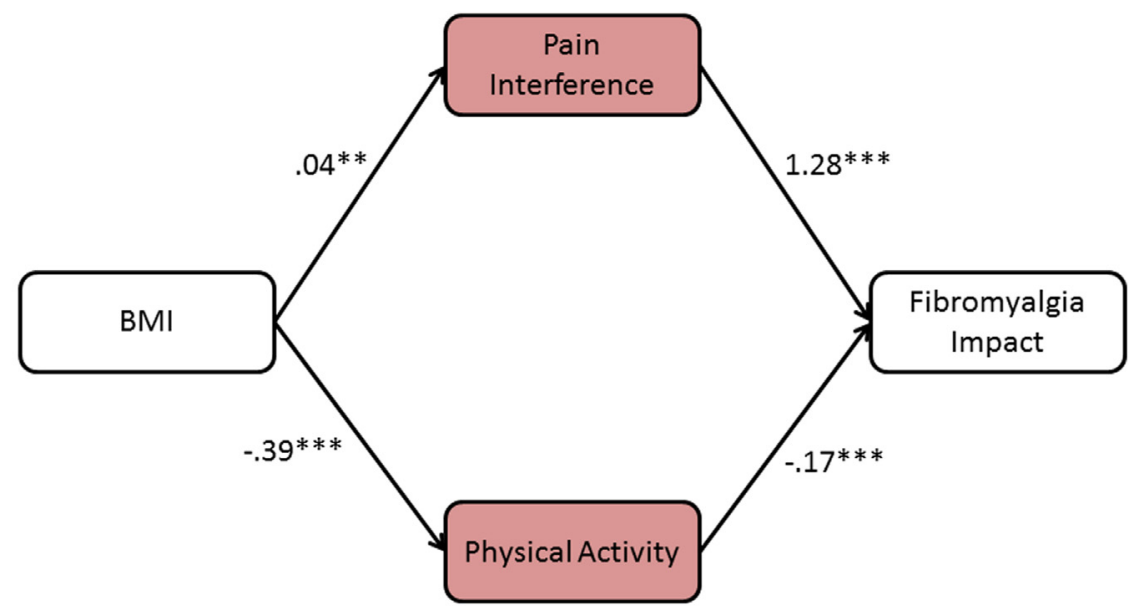

Unstandardized path coefficients. ${ }^{* *} \mathrm{p}<.01,{ }^{* * *} \mathrm{p}<.001$

Figure 2. Empirically derived model of body mass index (BMI), mechanisms, and fibromyalgia impact.

interrelationships of variables through mediation modeling. This strengthens our understanding of potential mechanisms. Second, the generalizability of our results is limited, given our clinical sample consisting of patients with moderate to severe symptoms. Our model will need to be validated in other clinical and community samples. Third, although anxiety and depression were not significant mediators in this model, it is important to point out that patients with fibromyalgia have a high prevalence of comorbid mood and anxiety disorders. These results were surprising, as literature suggests an association between mood and obesity $[34,35]$ and will need further study. Fourth, given that duration of symptoms was not assessed, we were unable to determine whether this could have influenced symptom burden. Fifth, because not all patients invited to participate returned completed surveys, participation bias could limit the generalizability of our findings. Finally, our sample size was large, as is necessary to obtain reliable results from structural equation modeling; however, large samples also have copious statistical power and hence can detect small associations that may not always be clinically meaningful. The present findings offer theoretical support for influence of BMI on fibromyalgia impact and putative mechanisms of action, but the clinical utility and meaningfulness of these modest interrelationships has yet to be determined in applied clinical settings.

\section{CONCLUSIONS}

To conclude, although a higher BMI increases pain and often serves as a barrier to physical activity, transcending this challenge even through the simplest of activities may translate to decreased pain. Obese and overweight patients with other chronic conditions are routinely told that they will help themselves if they move a little [36]. Our study is in consonance with these reports. Clinicians who encounter patients with fibromyalgia, particularly those with increased BMI, should invest time and resources in counseling patients to increase physical activity.

\section{ACKNOWLEDGMENTS}

Study data were collected and managed using REDCap electronic data capture tools hosted at Mayo Clinic. REDCap (Research Electronic Data Capture) is a secure, Web-based application designed to support data capture for research studies, providing the following: an intuitive interface for validated data entry; audit trails for tracking data manipulation and export procedures; automated export procedures for seamless data downloads to common statistical packages; and procedures for importing data from external sources.

\section{REFERENCES}

1. Yunus MB, Arslan S, Aldag JC. Relationship between body mass index and fibromyalgia features. Scand J Rheumatol 2002;31:27-31.

2. Kim C-H, Luedtke CA, Vincent A, Thompson JM, Oh TH. Association of body mass index with symptom severity and quality of life in patients with fibromyalgia. Arthritis Care Res 2012;64:222-228.

3. Ursini F, Naty S, Grembiale RD. Fibromyalgia and obesity: The hidden link. Rheumatol Int 2011;31:1403-1408.

4. Timmerman GM, Calfa NA, Stuifbergen AK. Correlates of body mass index in women with fibromyalgia. Orthop Nurs 2013;32:113-119.

5. Okifuji A, Donaldson GW, Barck L, Fine PG. Relationship between fibromyalgia and obesity in pain, function, mood, and sleep. J Pain 2010;11:1329-1337. 
6. Aparicio VA, Ortega FB, Carbonell-Baeza A, Camiletti D, Ruiz JR, DelgadoFernandez M. Relationship of weight status with mental and physical health in female fibromyalgia patients. Obes Facts 2011;4:443-448.

7. Neumann L, Lerner E, Glazer Y, Bolotin A, Shefer A, Buskila D. A crosssectional study of the relationship between body mass index and clinical characteristics, tenderness measures, quality of life, and physical functioning in fibromyalgia patients. Clin Rheumatol 2008;27: 1543-1547.

8. Baron RM, Kenny DA. The moderator-mediator variable distinction in social psychological research: Conceptual, strategic, and statistical considerations. J Personal Soc Psychol 1986;51:1173-1182.

9. Whipple M, McAllister S, Oh TH, Luedtke CA, Toussaint LL, Vincent A. Construction of a US fibromyalgia registry using the Fibromyalgia Research Survey Criteria. Clin Translat Sci 2013;6:398-399.

10. Wolfe F, Clauw DJ, Fitzcharles M-A, et al. Fibromyalgia criteria and severity scales for clinical and epidemiological studies: A modification of the ACR Preliminary Diagnostic Criteria for Fibromyalgia. J Rheumatol 2011;38:1113-1122.

11. Bennett RM, Friend R, Jones KD, Ward R, Han BK, Ross RL. The Revised Fibromyalgia Impact Questionnaire (FIQR): Validation and psychometric properties [Erratum appears in Arthritis Res Ther 2009;11:415]. Arthritis Res Ther 2009;11:R120.

12. Ware JE Jr. SF-36 Health Survey Update. Spine 2000;25:3130-3139.

13. Cleeland CS. The Brief Pain Inventory: User Guide. Houston, TX: Charles S. Cleeland; 2009.

14. McNair DM, Lorr M, Droppleman LF. EDITS Manual for the Profile of Mood States. San Diego, CA: Educational Testing Services; 1992.

15. Arnold LM, Hess EV, Hudson JI, Welge JA, Berno SE, Keck PE Jr. A randomized, placebo-controlled, double-blind, flexible-dose study of fluoxetine in the treatment of women with fibromyalgia. Am J Med 2002;112:191-197.

16. Williams DA, Arnold LM. Measures of fibromyalgia: Fibromyalgia Impact Questionnaire (FIQ), Brief Pain Inventory (BPI), Multidimensional Fatigue Inventory (MFI-20), Medical Outcomes Study (MOS) Sleep Scale, and Multiple Ability Self-Report Questionnaire (MASQ). Arthritis Care Res 2011;63(Suppl 11):S86-S97.

17. Arnold LM, Russell IJ, Diri EW, et al. A 14-week, randomized, doubleblinded, placebo-controlled monotherapy trial of pregabalin in patients with fibromyalgia [see Comment]. J Pain 2008;9:792-805.

18. Arnold LM, Gendreau RM, Palmer RH, Gendreau JF, Wang Y. Efficacy and safety of milnacipran $100 \mathrm{mg} /$ day in patients with fibromyalgia: Results of a randomized, double-blind, placebo-controlled trial. Arthritis Rheum 2010;62:2745-2756.

19. Da Costa D, Dobkin PL, Fitzcharles MA, et al. Determinants of health status in fibromyalgia: A comparative study with systemic lupus erythematosus. J Rheumatol 2000;27:365-372.

20. Neumann L, Berzak A, Buskila D. Measuring health status in Israeli patients with fibromyalgia syndrome and widespread pain and healthy individuals: Utility of the Short Form 36-Item Health Survey (SF-36). Semin Arthritis Rheum 2000;29:400-408.

21. Tuzun EH, Albayrak G, Eker L, Sozay S, Daskapan A. A comparison study of quality of life in women with fibromyalgia and myofascial pain syndrome. Disabil Rehabil 2004;26:198-202.
22. Arnold LM, Rosen A, Pritchett YL, et al. A randomized, double-blind, placebo-controlled trial of duloxetine in the treatment of women with fibromyalgia with or without major depressive disorder. Pain 2005; 119:5-15.

23. Chappell AS, Bradley LA, Wiltse C, Detke MJ, D'Souza DN, Spaeth M. A six-month double-blind, placebo-controlled, randomized clinical trial of duloxetine for the treatment of fibromyalgia. Int J Gen Med 2008;1: 91-102.

24. Mease PJ, Spaeth M, Clauw DJ, et al. Estimation of minimum clinically important difference for pain in fibromyalgia. Arthritis Care Res 2011; 63:821-826.

25. Russell IJ, Mease PJ, Smith TR, et al. Efficacy and safety of duloxetine for treatment of fibromyalgia in patients with or without major depressive disorder: Results from a 6-month, randomized, double-blind, placebocontrolled, fixed-dose trial. Pain 2008;136:432-444.

26. Bourgeois A, LeUnes A, Meyers M. Full-scale and short-form of the Profile of Mood States: A factor analytic comparison. J Sport Behav 2010;33:355-376.

27. Malin K, Littlejohn GO. Psychological control is a key modulator of fibromyalgia symptoms and comorbidities. J Pain Res 2012;5:463-471.

28. Katz RS, Heard AR, Mills M, Leavitt F. The prevalence and clinical impact of reported cognitive difficulties (fibrofog) in patients with rheumatic disease with and without fibromyalgia. J Clin Rheumatol 2004:10:53-58.

29. Kline RB. Principles and Practice of Structural Equation Modeling. New York, NY: Guilford Press; 2011.

30. Hayes AF. Introduction to Mediation, Moderation, and Conditional Process Analysis. New York: Guilford Press; 2013.

31. Hayes AF, Glynn CJ, Huge ME. Cautions regarding the interpretation of regression coefficients and hypothesis tests in linear models with interactions. Commun Methods Meas 2012;6:1-11.

32. Fontaine K, Conn L, Clauw D. Effects of lifestyle physical activity on perceived symptoms and physical function in adults with fibromyalgia: Results of a randomized trial. Arthritis Res Ther 2010;12:R55.

33. Levine JA, Vander Weg MW, Hill JO, Klesges RC. Non-exercise activity thermogenesis: The crouching tiger hidden dragon of societal weight gain. Arterioscler Thromb Vasc Biol 2006;26:729-736.

34. Johnston E, Johnson S, McLeod P, Johnston M. The relation of body mass index to depressive symptoms. Can J Public Health 2004;95:179-183.

35. Pasco JA, Williams LJ, Jacka FN, Brennan SL, Berk M. Obesity and the relationship with positive and negative affect. Aust N Z J Psychiatry 2013;47:477-482.

36. Penedo FJ, Dahn JR. Exercise and well-being: A review of mental and physical health benefits associated with physical activity. Curr Opin Psychiatry 2005;18:189-193.

This journal-based CME activity is designated for 1.0 AMA PRA Category 1 Credit $^{\mathrm{TM}}$ and can be completed online at www.me.aapmr.org. This activity is FREE to AAPMER members and available to non-members for a nominal fee. For assistance with claiming CME for this activity, please contact (847) 737-6000.

\section{CME Question}

Which is considered the most challenging symptom to treat with fibromyalgia patients?
a. sleep
b. depression
c. activity
d. anxiety

Answer online at me.aapmr.org 